\title{
mCRPC: neue Therapieoption für chemotherapienaive Patienten
}

- Mit dem Androgen-Biosynthese-Inhibitor Abirateronacetat (Zytiga ${ }^{\circledR}$ ) in Kombination mit Prednison oder Prednisolon kann Patienten mit metastasiertem kastrationsresistentem Prostatakarzinom (mCRPC), bei denen nach Versagen einer antihormonellen Behandlung eine Chemotherapie noch nicht indiziert ist, eine effektive und gut verträgliche Therapieoption angeboten werden, berichtete Prof. Peter Hammerer, Braunschweig. Aktuelle Studiendaten zeigen, dass die Substanz zu einer Verzögerung der Krankheits- und Beschwerdeprogression sowie zu einer Verlängerung des Überlebens führt.

In die randomisierte, kontrollierte Doppelblindstudie (COU-AA-302) wurden 1.088 asymptomatische oder mild symptomatische chemonaive mCRPC-Patienten aufgenom- men und mit Abirateronacetat $(1.000 \mathrm{mg} / \mathrm{d})$ plus Prednison (5 mg 2 x täglich) oder Placebo/Prednison behandelt. Abirateronacetat/ Prednison verlängerte das radiologisch bewertete progressionsfreie Überleben (Hazard Ratio [HR] 0,53; $\mathrm{p}<0,001)$ und das Gesamtüberleben (HR 0,75; $p=0,01)$ gegenüber Placebo [Ryan CJ et al. N Engl J Med 2013; 368: 138-48]. Zudem war die mediane Zeit bis zum Beginn der Chemotherapie ( 25,2 vs. 16,8 Monate, HR 0,58; $p<0,001$ ), bis zur Verschlechterung des ECOG-Status (12,3 vs. 10,9 Monate; HR 0,$82 ; p=0,005$ ) und bis zur PSA-Progression ( 11,1 vs. 5,6 Monate; HR 0,49; $p<0,001$ ) signifikant länger. Während die Patienten der Placebogruppe im Median nach 23,7 Monaten aufgrund krebsbedingter Schmerzen Opiate benötigten, war zum Auswertezeit- punkt im Verumarm noch kein Opiateinsatz erforderlich (HR 0,69; $p<0,001)$.

Die Ergebnisse dieser Studie führten zur Zulassungserweiterung von Abirateronacetat. Damit kann die Substanz jetzt nicht nur bei Männern eingesetzt werden, deren Erkrankung während oder nach einer Docetaxelhaltigen Chemotherapie progredient ist, sondern auch bereits bei nicht oder mild symptomatischen Männern, bei denen der Androgenentzug versagt und eine Chemotherapie noch nicht indiziert ist. Abdol A. Ameri

Launch-Pressekonferenz „EU-weite Zulassung von Zytiga ${ }^{\oplus}$ für die Behandlung Chemotherapie naiver Patienten mit mCRPC" München, 29. Januar 2013,

Veranstalter: Janssen-Cilag, Neuss

\section{Ejaculatio praecox: mehr als ein Lifestyle-Phänomen}

- Mit einer Prävalenz von bis zu $30 \%$ ist die Ejaculatio praecox (EP) weltweit die häufigste sexuelle Dysfunktion bei Männern unter 60 Jahren. Grundsätzlich werden nach Ausführung von Prof. Hartmut Porst, Hamburg, Präsident der Europäischen Gesellschaft für Sexualmedizin,, zwei Formen unterschieden, die lebenslange EP und die erworbene. Die schlimmste Form der EP ist die Ejaculatio ante portas, bei der es noch vor der Penetration zur Ejakulation kommt, sodass Betroffenen nicht in der Lage sind, Kinder zu zeugen.

„Der vorzeitige Samenerguss hat unglaublich negative Auswirkungen auf die Orgasmusfähigkeit und bedeutet einen enormen Stress für den Betroffenen und die Partnerin", erklärte Porst. Trotz seiner Häufigkeit ist die EP immer noch ein Tabuthema. Betroffene scheuen trotz des hohen Leidensducks den Weg zum Arzt. Häufig folgen dann ein Abbruch der sexuellen Beziehung oder sogar die Scheidung.

Pathogenetisch liegt der EP ein Störung der serotonergen Transporter zugrunde. Mit dem selektiven Serotonin-Wiederaufnahmehemmer (SSRI) Dapoxetin steht die einzig zugelassene Bedarfsmedikation der EP zur Verfügung, deren Wirksamkeit und Sicherheit auch in klinischen Studien dokumentiert ist. „Die Substanz Dapoxetin (Priligy ${ }^{\circledR}$ ) flutet schneller an und wird schneller eliminiert als andere SSRI, sie ist daher besonders als Bedarfsmedikation der EP geeignet", erklärte Dr. Thomas Kreutzig, Freiburg. Als Maß für die Wirksamkeit wurde in den Studien die intravaginale Ejakulationslatenzzeit (IELT), das heißt die Zeit zwischen Penetration und Ejakulation, verwendet. Bereits nach der ersten Dapoxetin-Gabe verlängerte sich dieser Zeitraum signifikant, was sich im Laufe der Zeit noch verstärkte. In einer zusammenfassenden Analyse der fünf Phase-III-Studien zeigte sich, dass sich die IELT durch die Therapie nach zwölf Wochen signifikant um mehr als das Dreifache verbesserte. Damit erhöhte sich auch die sexuelle Lebensqualität.

Dr. Susanne Kammerer

Pressegespräch „Wenn der Samenerguss in die Verlängerung geht: Zeitgemäße Impulse für die Behandlung der Ejaculatio praecox", Berlin, 21. Februar 2013

Veranstalter: Berlin-Chemie, Berlin

\section{Forschungspreis Prostatakarzinom}

— Bei der diesjährigen Jahrestagung der Deutschen Gesellschaft für Urologie (DGU) unterstützt Astellas mit dem „Forschungspreis Prostatakarzinom" wiederholt den wissenschaftlichen Nachwuchs aus Klinik und Praxis. Die mit 10.000 Euro dotierte Auszeichnung wird im Rahmen des 65. DGU-Kongresses (vom 25. bis 28. September 2013) in Dresden verliehen.

Bewerben können sich Wissenschaftler, bis zum einschließlich 40. Lebensjahr mit Forschungsstandort in Deutschland. Für die Teilnahme ist ein Projektplan zu erstellen, der Ziele, Methoden und Ressourcen sowie eine Zeitplanung und eine Zusammenfassung enthält. Das Forschungsprojekt darf noch nicht abgeschlossen oder veröffentlicht sein. Die Bewerbungsfrist für den „Forschungspreis Prostatakarzinom 2013“ endet am 1. Juni 2013. Informationen zu den Teilnahmebedingungen sind verfügbar auf der Internetseite der DGU (www.dgu-kongress.de/index.php?id=309) oder unter www.astellas.de/ueber_astellas/ forschung_entwicklung/forschungspreise. html.

Nach Informationen von Astellas, München 